\title{
Influência da Suplementação de Carboidrato na Função Imune de Judocas Durante o Treinamento
}

\author{
Influence of Carbohydrate Supplementation in the Immune Response of \\ Judoists During Training
}

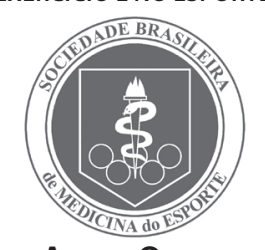

Artigo Original
Edmar Lacerda Mendes ${ }^{1}$

Ciro José Brito ${ }^{2}$

Ellencristina Silva Batista ${ }^{3}$

Carlos Henrique Osório Silva ${ }^{4}$

Sérgio Oliveira de Paula

Antonio José Natali ${ }^{6}$

1. Professor Substituto do

Departamento de Biologia Animal

da Universidade Federal de Viçosa,

Viçosa, MG.

2. Professor do Centro Universitário Metodista de Minas Izabela Hendrix, Belo Horizonte, MG.

3. Professora do Centro Universitário de Vila Velha, Vila Velha, ES.

4. Professor do Departamento de Informática, setor de Estatística, da Universidade Federal de Viçosa, Viçosa, MG.

5. Professor do Departamento de Biologia Animal da Universidade Federal de Viçosa, Viçosa, MG. 6. Professor do Departamento de Educação Física da Universidade Federal de Viçosa, Viçosa, MG.

Endereço para correspondência: Universidade Federal de Viçosa, Departamento de Educação Física

- A/C Prof. Antônio José Natali

- 36570-000 - Viçosa, MG.

Tel.: (31) 3899-4390

E-mail: anatali@ufv.br

Submetido em 07/12/2007

Versão final recebida em 24/04/2008

Aceito em 29/07/2008

\section{RESUMO}

O objetivo deste trabalho foi investigar os efeitos da suplementação de carboidrato, durante uma sessão de treino, sobre a função imune de atletas de judô. Dezesseis judocas do sexo masculino foram submetidos a duas sessões de treinamento de 120 minutos cada, com três dias de intervalo entre elas. Na primeira sessão, oito judocas, separados de forma aleatória, foram suplementados ( $3 \mathrm{~mL} / \mathrm{kg}$ peso corporal) com solução carboidratada (grupo CHO) e os demais, com solução placebo (grupo PLA), de forma duplo-cega. Na segunda sessão os tratamentos foram invertidos. O número de leucócitos, linfócitos, monócitos, eosinófilos, neutrófilos, os níveis de cortisol e as concentrações de glicose e lactato foram medidos em repouso (Pré-E), imediatamente após (Pós-E) e uma hora após o término da sessão de treino (1h pós-E). Os resultados mostraram que a glicemia aumentou $(p<0,05)$ durante a sessão de treino no grupo $\mathrm{CHO}$ e reduziu-se $(p<0,05)$ no grupo PLA. O cortisol aumentou $(p<0,05)$ durante a sessão de treino, independente do tipo de solução consumida, bem como durante a recuperação no grupo PLA. O consumo de CHO resultou em menor $(p<0,05)$ leucocitose, quando comparado com o PLA, nos períodos Pós-E e $1 \mathrm{~h}$ Pós-E. A elevação da concentração de lactato sangüíneo decorrente do exercício correlacionou-se positivamente com o aumento dos leucócitos $(r=0,86, p<0,001)$ nos dois grupos. Concluiu-se que a ingestão de bebida carboidratada por atletas de judô durante uma sessão de treino resultou em menor perturbação da contagem total de leucócitos e suas subclasses: linfócitos, monócitos, eosinófilos e neutrófilos. Esses resultados sugerem proteção à saúde imunológica de judocas fomentada por essa estratégia nutricional.

Palavras-chave: judô, treinamento, ciências da nutrição, sistema imune.

\begin{abstract}
The aim of this study was to investigate the effects of carbohydrate supplementation on the immunological function of judoists during a training session. Sixteen male judo athletes were submitted to two 120 min training sessions within 3 days interval. In the first session eight athletes randomly chosen were supplemented ( $3 \mathrm{~mL} / \mathrm{kg}$ body weight) with a carbohydrate solution ( $\mathrm{CHO}$ group) while the others ingested a placebo solution (PLA group) in a randomized and double blind way. In the second session, treatments were inverted. Leukocyte, lymphocyte, monocyte, eosinophil and neutrophil were counted and blood levels of cortisol, glucose and lactate were assessed at rest (Pre-E), immediately after the training session (Post-E) and one hour after the training session (1h Post-E). The results showed that the blood glucose levels increased during the training session when athletes were given $\mathrm{CHO}$ and decreased when they ingested PLA. Cortisol levels increased during the training session in both conditions and decreased $1 \mathrm{~h}$ Post-E. Athletes consuming $\mathrm{CHO}$ presented a reduced number of leukocytes when compared to those ingesting PLA in both Post-E and $1 \mathrm{~h}$ Post-E periods. The increase in blood lactate concentration in response to exercise training was positively correlated with the increase in the leukocyte number $(r=0.86, p<0.001)$ in both conditions. It was concluded that $\mathrm{CHO}$ supplementation during a judo training session resulted in less disturbance of total leukocytes. It suggests that such nutritional strategy protected the athletes' immunological health.
\end{abstract}

Keywords: judo, training, nutritional sciences, immune system.

\section{INTRODUÇÃO}

O exercício intenso prolongado altera a imunidade em função do estresse fisiológico e, conseqüentemente, causa imunodepressão ${ }^{(1,2)}$. Por exemplo, imediatamente após exercício com intensidade de 70 a 85\% do consumo máximo de oxigênio $\left(\mathrm{VO}_{2 \mathrm{máx}}\right)$, os leucócitos totais aumentaram de 50 a 100\% na corrente sanguínea. Após 30 minutos de recuperação houve declínio da contagem do número de leucócitos para 30 a 60\% abaixo dos níveis de repouso, permanecendo assim por três a seis horas. Porém, em exercício moderado, com $50 \%$ do $\mathrm{VO}_{2 \text { máx }}$ a contagem do número de leucócitos não sofreu declínio durante a recuperaçãa(3).

A imunodepressão em resposta ao exercício envolve diversos mecanismos, tais como a liberação de hormônios estressores, a alteração da temperatura corporal, o aumento do fluxo sanguíneo e a desidratação ${ }^{(4)}$. Todavia, durante o exercício prolongado as respostas de hormônios estressores podem ser atenuadas pela ingestão de carboidrato $(\mathrm{CHO})$, provavelmente pela maior concentração de glicose sanguínea ${ }^{(1)}$. A ingestão de $\mathrm{CHO}$ durante o exercício de resistência aeróbica tem sido associada a fatores tais como: redução das respostas do cortisol, menor perturbação da contagem do número de células do sistema imune no sangue e diminuição da atividade fagocitária e oxidativa dos granulócitos e monócitos ${ }^{(4,5)}$.

Contudo, nas modalidades de luta, como o judô, caracterizado por picos de atividade anaeróbica lática ${ }^{(6,7)}$, as respostas do sistema imune são pouco conhecidas, assim como os efeitos da ingestão de $\mathrm{CHO}$ sobre essas respostas. O presente estudo teve como objetivo, portanto, investigar os efeitos da suplementação de carboidrato, durante uma sessão de treino, sobre a função imune de atletas de judô. 


\section{MÉTODOS}

Amostra: Participaram deste estudo 16 judocas masculinos com idade de 24,06 $\pm 2,59$ anos (média \pm desvio padrão), peso corporal de $76,78 \pm 9,42 \mathrm{~kg}$ e IMC de 24,45 \pm 2,45kg/m², filiados à Liga Mineira de Judô pela Associação Atlética Acadêmica da Universidade Federal de Viçosa (AAA-UFV). Este estudo foi previamente aprovado pelo Comitê de Ética em Pesquisas com Seres Humanos da Universidade Federal de Viçosa e os sujeitos assinaram o termo de consentimento livre e esclarecido.

Desenho experimental: Os sujeitos foram submetidos a duas sessões de treino de judô, com três dias de intervalo entre elas. Na primeira sessão, oito atletas foram escolhidos aleatoriamente para consumir solução carboidratada comercial, contendo 6\% de carboidrato (Grupo CHO). Os demais atletas consumiram solução placebo (0\% de carboidrato, Grupo PLA). Os tratamentos foram invertidos na segunda sessão.

Sessão de treino: Cada sessão de treino durou 120 minutos, sendo 40 minutos de ginástica localizada, 40 minutos de técnica e 40 minutos de lutas. Os atletas repousaram 60 minutos no dojô após o final do treino.

Procedimentos: Os atletas foram orientados a não treinar no dia anterior ao do teste e a manter jejum nas oito horas que antecederam as coletas de dados. Os procedimentos experimentais iniciaram-se às sete horas da manhã, no local de treinamento, com a primeira coleta de sangue. Logo após foi oferecido o desjejum individual, atendendo-se ao resultado da avaliação nutricional de cada atleta. Uma hora após o desjejum os atletas iniciaram a sessão de treino. Imediatamente após o término da sessão foram coletadas novas amostras de sangue. Esse procedimento foi repetido uma hora após o término da sessão.

Coleta de sangue e análises: Amostras de sangue ( $5 \mathrm{ml}$ ) foram coletadas de uma veia antecubital em tubos contendo K3 EDTA e 0,3ml de heparina (BD Vacutainer ${ }^{\oplus}$ ) e centrifugadas a 3.400rpm por 10 minutos para separar o plasma. Os leucócitos totais e suas subclasses foram analisados em triplicata usando-se um analisador hematológico automatizado (Horiba ABX, São Paulo, SP, Brasil). O cortisol foi mensurado utilizando-se kit comercial de radioimunoensaio (Incstar - Stillwater, MN, EUA). Nas mesmas ocasiões dessas coletas, foram extraídas duas microamostras sangüíneas $(25 \mu l)$ da polpa digital para monitoração da glicemia e da concentração de lactato sanguíneas. Foram utilizados os aparelhos portáteis Accutrend Lactate e Accu-Check Advantage (Roche, Mannheim, Alemanha) para a análise da glicemia e concentração de lactato, respectivamente.

Refeição pré-exercício: Após avaliação dos hábitos alimentares, por meio de questionário e de recordatório habitual da dieta, calculou-se a dieta individualizada para os atletas consumirem nos três dias anteriores ao início do experimento ${ }^{(8-10)}$. O nível de atividade física individual foi obtido pelo International Physical Activity Questionary (IPAQ). O teor energético da dieta atendia de 90 a 110\% da necessidade estimada de energia (estimate energy requeriment - EER). O desjejum foi padronizado como a refeição préexercício atendendo a 14\% de energia da EER de cada atleta, sendo composto por suco industrializado, pão de forma, requeijão cremoso e maçã. As dietas foram calculadas utilizando-se o programa Diet-Pro versão 4.0(11).

Composição das soluções e protocolo de hidratação: Utilizou-se como solução carboidratada um repositor hidroeletrolítico comercial (Gatorade ${ }^{\varpi}$ ) com a seguinte composição: carboidrato (sacarose e frutose), $6 \mathrm{~g} / 100 \mathrm{ml}$; sódio, 45mg/100ml; potássio, 12mg/100ml; cloreto, 42mg/ $100 \mathrm{ml}$. Para a solução placebo foi utilizado preparado sólido para refresco de baixa caloria (Clight ${ }^{\circledR}$ ), sabor tangerina, com cor, sabor e textura idênticos aos da solução carboidratada, com a seguinte composição: sódio, $87 \mathrm{mg} / 100 \mathrm{ml}$; cloreto, $80 \mathrm{mg} / 100 \mathrm{ml}$ ). Cada atleta consumiu $3 \mathrm{ml}$ por quilo de peso corporal de solução a cada 15 minutos. Os atletas se hidrataram nos minutos $0,15,30,45,60,75,90$ e 120 do treinamento e nos minutos 135, 150, 165 e 180 após (recuperação), de forma duplo-cega ${ }^{(12)}$.

Análise estatística: Inicialmente, adotou-se o seguinte modelo misto e procedeu-se à ANOVA dos dados, considerando-se a estrutura de medidas repetidas no mesmo atleta, com inferências usuais pelo teste $F$,

$$
D_{i j k}=\mu+T_{i}+P_{j}+A_{k}+\varepsilon_{i j k}
$$

em que $D_{i j k}$ é a diferença entre os valores final e inicial (entre Pré-E e Pós-E; ou 1 h Pós-E e Pós-E) calculada para todos os atletas, $\mu$ é a média geral, $T_{i}$ para $i=1,2$ é o efeito fixo dos dois tratamentos (solução placebo e bebida carboidratada); $P_{j}$ para $j=1,2$ é o efeito fixo do período experimental, $A_{k}$ para $k=1,2, \ldots, 16$ é o efeito aleatório do atleta e $\varepsilon_{i j k}$ é o erro aleatório não observável do modelo com as usuais pressuposições de normalidade e independência. As hipóteses testadas foram de igualdade entre médias para os efeitos fixos e variabilidade nula para os efeitos aleatórios. Aplicou-se o teste $t$ de Student (dados pareados) para verificar diferenças significativas entre os valores Pré-E e Pós-E e também entre os valores $1 \mathrm{~h}$ Pós-E e Pós-E. Foi realizado o teste t para amostras independentes quando o objetivo era comparar os dois tratamentos (solução placebo vs. carboidratada) em um mesmo tempo (Pré-E, Pós-E e 1h PósE) de avaliação. O teste não paramétrico de Wilcoxon foi utilizado como um auxílio adicional nas conclusões. A pressuposição de normalidade do teste $t$ para dados pareados foi verificada pelo teste de Shapiro-Wilk. Para estudar a associação das variáveis lactato vs. leucócito realizou-se a análise de regressão linear simples. Os dados foram analisados usando-se o software SPSS (Statistical Package for Social Sciences), versão 12 para Windows, com o auxílio do pacote computacional SAS (Statistical Analysis System, SAS Institute Inc., Cary, NC, EUA - versão 8.1). Adotou-se o nível de significância de até $5 \%$ em todos os procedimentos.

\section{RESULTADOS}

A glicemia aumentou significativamente entre Pré-E e Pós-E (95,31 mg/ dl vs. $117,39 \mathrm{mg} / \mathrm{dl}$, respectivamente, $\mathrm{p}$-valor $=0,003)$ e reduziu-se significativamente entre Pós-E e 1 h Pós-E (117,39mg/dl vs. 94,44mg/dl, respectivamente, $\mathrm{p}$-valor $=0,002)$ no grupo que consumiu $\mathrm{CHO}$ e reduziu-se significativamente entre Pré-E e Pós-E $(94,44 \mathrm{mg} / \mathrm{dl}$ vs. 88,06mg/dl, respectivamente, $p$-valor $=0,045)$ no grupo PLA. Houve diferença significativa entre os grupos CHO e PLA no período Pós-E (117,39mg/dl vs. 88,06, respectivamente, $\mathrm{p}$-valor $=0,025)$, como demonstrado na figura 1 .

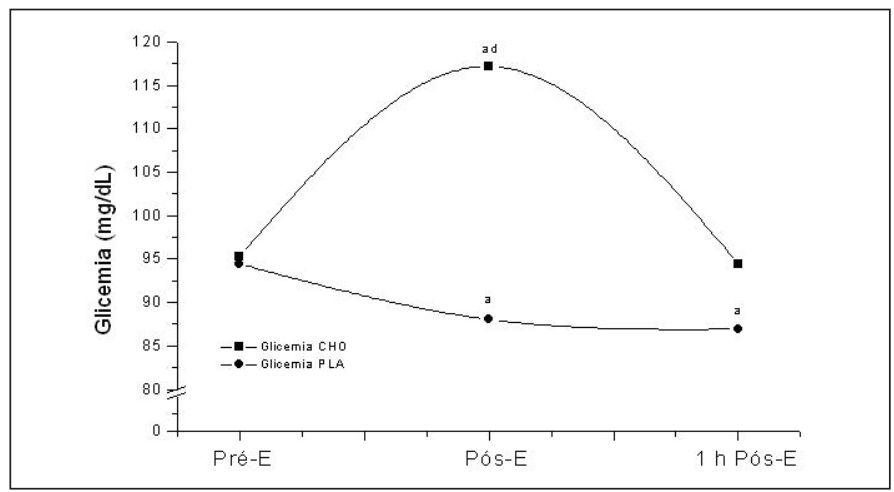

Figura 1. Concentração média da glicemia sanguínea durante o treino de judô. a = diferença significativa entre médias Pré-E vs. Pós-E e Pós-E vs. 1 h Pós-E $(p<0,05)$ para o grupo que ingeriu carboidrato e placebo. ${ }^{\mathrm{d}}=$ diferença significativa entre soluções placebo e carboidrato dentro do mesmo período experimental $(p<0,05)$.

A figura 2 apresenta os valores médios das (ou perfis dos atletas para as) concentrações de cortisol durante 120 minutos de treino, seguidos de 60 minutos de recuperação.

O cortisol aumentou significativamente durante os 120 min de exercício, do Pré-E para o Pós-E, independente do tipo de solução consumida $(11,46 \mathrm{mg} / \mathrm{dl}$ vs. 13,99mg/dl, respectivamente, $\mathrm{p}$-valor $=0,02$ para $\mathrm{CHO}$ e $10,95 \mathrm{mg} / \mathrm{dl}$ vs. $13,95 \mathrm{mg} / \mathrm{dl}$, respectivamente, $\mathrm{p}$-valor = 0,03 para PLA). Durante a recuperação (entre 120 e 180 min), também ocorreu elevação do cortisol, porém, esta só foi significativa nos atletas que consumiram o placebo (13,95mg/dl vs. 16,87mg/dl, respectivamente, $\mathrm{p}$-valor =0,04).

A concentração de leucócitos e suas subclasses avaliadas antes, imediatamente após e uma hora após a sessão de treinamento em judô, frente à ingestão de carboidrato ou placebo, são apresentados na tabela 1.

O consumo de carboidrato resultou em menor leucocitose ( $p$-valor $<0,05)$, se comparado com o do placebo, nos períodos Pós-E e $1 \mathrm{~h}$ 


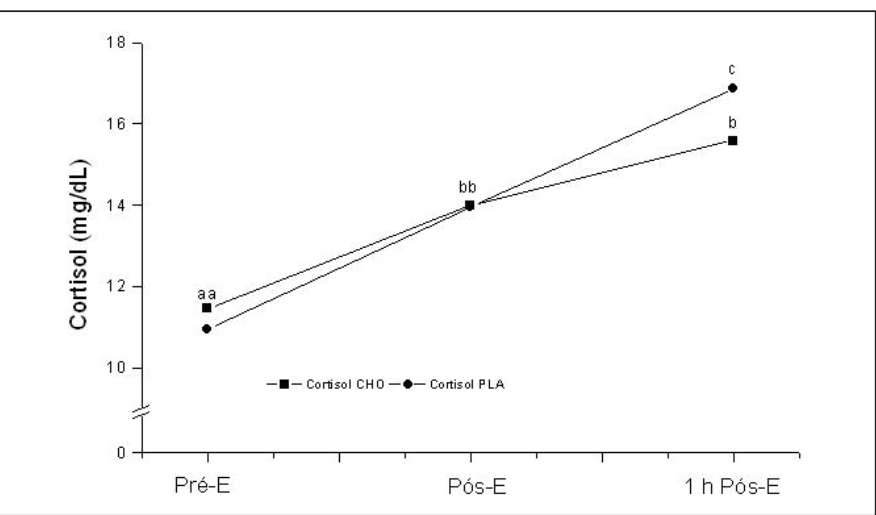

Figura 2. Concentração média do cortisol durante o treino de judô com respectivas comparações entre os tratamentos (carboidrato vs. placebo) em cada período de medição. Letras iguais indicam diferença não significativa ( $p$-valor $>0,05$ ).

$a, b, c=$ diferença significativa entre médias Pré-E vs. Pós-E e Pós-E v.s 1 h Pós-E $(p<0,05)$ para o grupo que ingeriu carboidrato e placebo.

Tabela 1. Concentrações (média \pm desvio padrão) de leucócitos, linfócitos, monócitos, eosinófilos e neutrófilos antes (Pré-E), imediatamente após (Pós-E) e uma hora após (1h Pós-E) a sessão de treino de judô com ingestão de solução carboidratada $(\mathrm{CHO})$ e placebo $(\mathrm{PLA})$

\begin{tabular}{c|c|c|c|c}
\hline & & \multicolumn{3}{|c}{ Período de avaliação } \\
\hline \multirow{2}{*}{ Variáveis } & Solução & Pré-E & Pós-E & 1h Pós-E \\
\hline \multirow{2}{*}{ Leucócitos $^{(1)}$} & CHO & $7.456 \pm 1.850$ & $8.481 \pm 3.100$ & $8.375 \pm 3.211$ \\
\cline { 2 - 5 } & PLA & $7.456 \pm 1.820^{\mathrm{a}}$ & $8.856 \pm 2.876^{\mathrm{b}, \mathrm{d}}$ & $8.800 \pm 2.814^{\mathrm{a}, \mathrm{b}, \mathrm{d}}$ \\
\hline \multirow{2}{*}{ Linfócitos $^{(1)}$} & $\mathrm{CHO}$ & $2.926 \pm 66$ & $2.350 \pm 110$ & $2.110 \pm 96$ \\
\cline { 2 - 5 } & $\mathrm{PLA}$ & $3.078 \pm 81^{\mathrm{a}}$ & $2.248 \pm 109^{\mathrm{b}}$ & $1.844 \pm 108^{\mathrm{c}}$ \\
\hline \multirow{2}{*}{ Monócitos $^{(1)}$} & $\mathrm{CHO}$ & $413 \pm 98$ & $369 \pm 106$ & $326 \pm 92$ \\
\cline { 2 - 5 } & $\mathrm{PLA}$ & $207 \pm 66$ & $215 \pm 100$ & $209 \pm 96^{\mathrm{a}}$ \\
\hline \multirow{2}{*}{ Eosinófilos $^{(1)}$} & $\mathrm{CHO}$ & $391 \pm 104$ & $403 \pm 120$ & $385 \pm 122$ \\
\cline { 2 - 5 } & $\mathrm{PLA}$ & $221 \pm 81$ & $278 \pm 89^{\mathrm{a}}$ & $295 \pm 108^{\mathrm{a}}$ \\
\hline \multirow{2}{*}{ Neutrófilos(1) $^{(1)}$} & $\mathrm{CHO}$ & $3.758 \pm 1.379$ & $4.969 \pm 2.183^{\mathrm{a}, \mathrm{d}}$ & $5.247 \pm 2.791^{\mathrm{a}, \mathrm{d}}$ \\
\cline { 2 - 5 } & $\mathrm{PLA}$ & $3.917 \pm 1.386$ & $6.258 \pm 2.698^{\mathrm{a}}$ & $6.754 \pm 2.791^{\mathrm{a}}$ \\
\hline
\end{tabular}

- concentração/ $/ \mathrm{mm}^{3}, \mathrm{a}, \mathrm{b}, \mathrm{c}=$ diferença significativa ao longo do tempo para a mesma solução $(p$-valor $<0,05),{ }^{d}=$ diferença significativa entre tratamentos ( $p$-valor $\left.<0,05\right)$, dentro de um mesmo período de avaliação.

pós-E. Apesar da redução observada na concentração de linfócitos, o consumo de $\mathrm{CHO}$ apresentou efeito protetor, pois não houve diferença significativa entre os valores de linfócitos observados antes do exercício (Pré-E), imediatamente após o treinamento (Pós-E) e 60 minutos após o exercício (1h Pós-E). Por outro lado, os atletas que consumiram a solução PLA apresentaram queda significativa de linfócitos no período Pós-E e 1 h Pós-E, em relação aos valores Pré-E ( $p$-valor < 0,05).

Independente da solução consumida, não houve diferença significativa entre as concentrações Pré-E e Pós-E de monócitos ( $p$-valor > 0,05). Entretanto, da mesma forma, o consumo de $\mathrm{CHO}$ apresentou efeito protetor, pois não ocorreu redução significativa nas concentrações de monócitos nos períodos Pós-E e 1h Pós-E ( $p$-valor < 0,05).

Em relação aos eosinófilos, observou-se aumento entre os níveis Pré-E e Pós-E em ambos os grupos. Entretanto, esse aumento só foi significativo para o grupo que consumiu PLA ( $p$-valor $<0,05$ ). Além disso, sua concentração continuou elevando-se 1h Pós-E no grupo PLA, apesar de não ter sido significativa em relação ao período Pós-E ( $p$-valor > 0,05).

Houve aumento significativo na concentração dos neutrófilos nos períodos Pré-E e Pós-E ( $p$-valor < 0,05) em ambos os grupos, independente da solução consumida. Entre os períodos Pós-E e $1 \mathrm{~h}$ Pós-E também ocorreu aumento na concentração de neutrófilos nos dois grupos. Entretanto, esse aumento não foi significativo ( $p$-valor $>0,05$ ). Apesar do aumento significativo observado entre os períodos Pré-E e Pós-E para ambas as soluções, a magnitude do aumento dos neutrófilos verificado no grupo PLA foi significativamente superior ao ocorrido frente ao consumo de $\mathrm{CHO}$, tanto no período Pós-E quanto no 1 h Pós-E (p-valor <0,05).

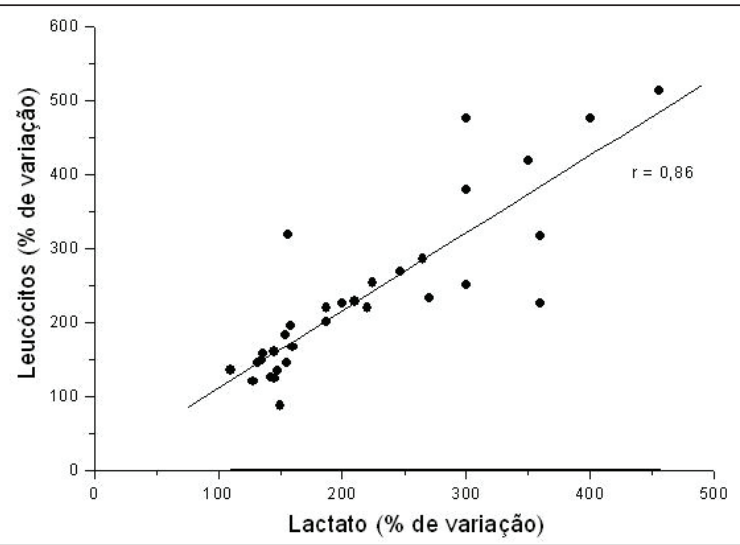

Figura 3. Dispersão e reta de regressão linear simples correspondentes ao percentual de variação do lactato sanguíneo e do número de leucócitos nos momentos 0 e 120 minutos dos atletas avaliados nas duas sessões de treino de judô.

A figura 3 apresenta a dispersão dos valores do lactato sanguíneo e dos níveis de leucócitos nos momentos 0 e 120 minutos nos dois grupos.

Os pontos dispersos na figura 3 demonstram que a elevação do lactato decorrente do exercício correlacionou-se positivamente com o aumento dos leucócitos ( $r=0,86, p$-valor $<0,001)$.

\section{DISCUSSÃO}

A ingestão de $\mathrm{CHO}$ durante o treino resultou em maior disponibilidade de glicose na corrente sanguínea. Entretanto, não foi capaz de inibir a expressão de cortisol, se comparado com o PLA. Todavia, durante o período de recuperação, o grupo $\mathrm{CHO}$ apresentou melhor resposta em conter a expressão de cortisol, em relação ao grupo PLA, indicando possível associação entre consumo de solução carboidratada e menor perturbação do eixo hipotálamo-pituitária-adrenal ( EHPA). O presente estudo demonstrou que o consumo de carboidratos resultou em menor leucocitose ao final do treino e uma hora após o término deste.

Nosso estudo demonstrou que o consumo de carboidratos atenua a resposta do cortisol no pós-exercício (figura 2). Um dos fatores que aumenta a liberação de cortisol é a baixa da glicose na corrente sanguínea. Dessa forma, o suprimento exógeno de carboidratos durante o exercício resulta em menor demanda dos estoques endógenos de energia, o que, por sua vez, tende a diminuir a liberação de hormônios catabólicos como o cortisol|(13).

$\mathrm{Na}$ ação nutricional que antecedeu o experimento, a dieta prescrita continha dose substancial de carboidrato, específica ao peso corporal (1g. kg peso corporal ${ }^{-1}$ ), para manter a glicemia sanguínea e prevenir a hipoglicemia dos atletas no grupo PLA. Isso pode ter resultado em resposta imune e hormonal similar à observada após os 120 min de exercício em ambos os tratamentos no presente estudo. Sabe-se que o exercício estimula o EHPA, o que resulta na elevação dos níveis de cortisol plasmático ${ }^{(13)}$. Em adição ao estímulo do exercício, os níveis de cortisol são elevados em resposta à redução dos níveis de glicose sanguínea ${ }^{(14)}$. A ingestão de $\mathrm{CHO}$ aumenta a disponibilidade de glicose ${ }^{(1)}$, conseqüentemente, a ingestão de CHO exógeno durante o exercício pode influenciar a resposta imune pela manutenção dos níveis de glicose sanguínea, reduzindo, assim, a produção de cortisol ${ }^{(1,14)}$. Embora a ingestão de carboidrato tenha demonstrado reduzir as perturbações do sistema imune em esportes de resistência(15), no judô ainda não existem relatos científicos sobre tal fato.

A menor liberação de cortisol resulta em menor ativação do EPHA e, por conseqüência, menor perturbação do sistema imune ${ }^{(13)}$, uma vez que os glicocorticóides tendem a induzir apoptose de células imunes ${ }^{(16)}$. Os resultados deste estudo estão de acordo com os de outros em que foi observada menor concentração plasmática de cortisol em indivíduos corredores e ciclistas que consumiram carboidratos ${ }^{(2,16)}$, apesar das diferenças entre as modalidades.

Diversos estudos demonstraram associação direta entre o exercício de longa duração e a imunodepressão ${ }^{(2,3)}$. O aumento na concentração de leucócitos e suas subpopulações tem sido observado em estudos 
envolvendo exercício de resistência aeróbica de longa duração. Por exemplo, Green et al.(17) observaram aumento superior a 100\% nas concentrações de leucócitos após 2,5 horas de ciclismo a 85\% do limiar anaeróbico, sendo que as concentrações continuaram a se elevar uma hora após o término das atividades. Natale et al. ${ }^{(18)}$ verificaram, em três diferentes modelos de exercícios (1 - cinco minutos de ciclismo entre 90 e $97 \%$ do $\mathrm{VO}_{2 \text { máxi }} 2$ - duas horas de ciclismo a $60 \%$ do $\mathrm{VO}_{2 \text { máxi }} 3$ - circuito de força com três séries de 10 repetições entre 60 e $70 \%$ de uma repetição máxima - 1RM), que duas horas de exercício aeróbico causaram maior leucocitose em relação ao exercício de força.

Concomitantemente ao aumento dos leucócitos plasmáticos (tabela 3), observou-se também maior concentração sanguínea de cortisol (figura 2), evidenciando, assim, a influência de hormônios estressores em perturbações no sistema imunológico. Apesar de os resultados observados aqui estarem de acordo com os de outros estudos ${ }^{(19,20)}$, a influência desses hormônios na contagem de células sanguíneas não está totalmente esclarecida (21). No presente estudo, apesar de ser observada menor leucocitose ao final do exercício no grupo CHO, as concentrações de cortisol não diferiram entre os grupos CHO e PLA. Opstad et al.(19) observaram que os níveis de cortisol se elevam durante o ciclismo de longa duração, entretanto, a influência dos hormônios na contagem de células, provavelmente, está associada à interação entre corticosteróides (cortisol) e catecolaminas (adrenalina e noradrenalina).

Além dos hormônios catabólicos, a produção excessiva de lactato pode perturbar o sistema imunológico. O aumento na produção de lactato observado neste estudo é resultado da alta intensidade do exercício, o que era esperado, uma vez que o judô é caracterizado por picos de atividade onde predomina o metabolismo anaeróbico láctico(6). Isso demonstra que não somente a duração, mas também a intensidade do exercício pode contribuir para a leucocitose durante uma sessão de treino de judô. Esses achados corroboram os dos estudos de Nemet et al.(22) em lutadores, onde observaram alta correlação $(r=0,74)$ entre a concentração de lactato sanguíneo e a leucocitose.

Ao final da sessão de treino ocorreu redução na concentração de linfócitos, independente da solução consumida, entretanto, essa só foi significativa para o grupo PLA. A redução na concentração de linfócitos aumenta a predisposição a infecções(23). Os resultados obtidos neste estudo estão de acordo com os de outros, onde se observou um efeito protetor dos carboidratos sobre a concentração de linfócitos ${ }^{(23,24)}$. Lancaster et al.(24) observaram que o consumo de carboidratos durante 2,5 horas de exercício preveniu o decréscimo na concentração de linfócitos, em relação ao consumo de placebo. Por outro lado, Nieman et al. ${ }^{(25)}$ observaram menor concentração de linfócitos após três horas de corrida no grupo que consumiu carboidratos, em relação ao grupo placebo.

\section{REFERÊNCIAS BIBLIOGRÁFICAS}

. Bishop NC, Blannin AK, Robson PJ, Walsh NP, Gleeson M. The effects of carbohydrate supplementation on immune responses to a soccer-specific exercise protocol. J Sports Sci. 1999;17(10):787-96.

2. Nieman DC, Henson DA, Smith LL, Utter AC, Vinci DM, Davis JM, et al. Cytokine changes after a marathon race. J Appl Physiol. 2001;91(1):109-14.

3. Pedersen BK, Rohde T, Ostrowski K. Recovery of the immune system after exercise. Acta Physiol Scand. 1998;162(3):325-32.

4. Nieman DC. Immune response to heavy exertion. J Appl Physiol. 1997:82(5):1385-94.

5. Nieman DC. Current perspective on exercise immunology. Curr Sports Med Rep. 2003;2(5):239-42.

6. Franchine E. Judô: Desempenho competitivo. 1ª ed Manole: S Paulo., 2001.

7. Thorland WG, Tipton CM, Lohman TG, Bowers RW, Housh TJ, Johnson GO, et al. Midwest wrestling study: prediction of minimal weight for high school wrestlers. Med Sci Sports Exerc. 1991;23(9):1102-10.

8. Institute of Medicine. Dietary References Intakes for energy, carbohydrate, fiber, fat, fatty acids, cholesterol, protein and amino acids. Washington, DC: The National Academy Press. 2002:936

9. Institute of Medicine. Dietary Reference Intakes for Vitamin C, Vitamin E, Selenium, and Carotenoids. Food and Nutrition Board. Institute of Medicine. 2000.

10. Sociedade Brasileira de Cardiologia. III Diretrizes Brasileiras Sobre Dislipidemias e Diretrizes de Prevenção da Aterosclerose do Departamento de Aterosclerose da Sociedade Brasileira de Cardiologia. Arquivos Brasileiros de Cardiologia. 2001;77(3):1-48

11. Monterio J, Esteves, EA, Mafia, UCC. Diet Pro versão 4.0. Agromidia, Viçosa. 2001.

12. Brito C. Hidratação com e sem carboidratos durante o treinamento de judô. Dissertação de Mestrado Programa de Pós-graduação em Ciência da Nutrição, Universidade Federal de Viçosa. 2005.

13. Mitchell JB, Costill DL, Houmard JA, Flynn MG, Fink WJ, Beltz JD. Influence of carbohydrate ingestion on counterregulatory hormones during prolonged exercise. Int J Sports Med. 1990;11(1):33-6.

14. Nieman DC. Influence of carbohydrate on the immune response to intensive, prolonged exercise. Exerc Immunol Rev. 1998;4:64-76.

15. Nieman DC, Davis JM, Brown VA, Henson DA, Dumke CL, Utter AC, et al. Influence of carbohydrate ingestion on immune changes after $2 \mathrm{~h}$ of intensive resistance training. J Appl Physiol. 2004;96(4):1292-8.
Os níveis de monócitos reduziram-se significativamente após o exercício. Entretanto, não houve diferença entre o grupo que ingeriu $\mathrm{CHO}$ ou PLA. Após o exercício, o consumo de carboidratos exerceu efeito protetor, em relação à redução dos números de monócitos, o que não foi observado no grupo PLA. Os monócitos são células de defesa que migram pelo sangue e se diferenciam em macrófagos nas células-alvo(26). Assim como observado no presente estudo, o exercício de longa duração tem sido associado à elevação na concentração de monócitos no plasma ${ }^{(27)}$ Por outro lado, o consumo de carboidratos durante o exercício tende a reduzir as concentrações plasmáticas de monócitos ${ }^{(15,27)}$.

O aumento significativo nas concentrações de eosinófilos foi observado somente no grupo que ingeriu PLA. Essas células sanguíneas são capazes de fagocitar corpos estranhos e sua presença no plasma em grandes concentrações está relacionada a ocorrência de infecções. A ingestão de carboidratos pode reduzir a perturbação dessas células, estando o mecanismo condicionado à expressão de cortisol plasmático(18).

A concentração de neutrófilos aumentou significativamente entre 0 e 120 minutos, independente do tipo de solução consumida. Entretanto, as concentrações observadas no grupo CHO foram significativamente menores em relação ao grupo PLA. Os neutrófilos são os principais fagócitos do sangue e participam de reações inflamatórias. No presente estudo, houve aumento nas concentrações plasmáticas de neutrófilos, corroborando os achados de Henson et al.(27), que avaliaram exercício de longa duração. O consumo de carboidratos demonstrou efeito protetor, resultando em menores concentrações de neutrófilos.

Concluiu-se que a ingestão de bebida carboidratada por atletas de judô durante uma sessão de treino resultou em maiores concentrações de glicose sanguínea, menores concentrações de cortisol e menor perturbação da contagem total de leucócitos e suas subclasses: linfócitos, monócitos, eosinófilos e neutrófilos. Além disso, as concentrações sanguíneas de lactato aumentaram significativamente entre o início e o final do exercício, independente do tipo de solução consumida, correlacionando-se positivamente com a leucocitose durante a sessão de treino. Esses resultados sugerem ação protetora à saúde imunológica de judocas fomentada por essa estratégia nutricional.

\section{AGRADECIMENTOS}

À equipe de judô da LUVE, pela colaboração incondicional na condução do experimento.

Aos Departamentos de Nutrição e Saúde, Educação Física e Biologia Animal da Universidade Federal de Viçosa.

Todos os autores declararam não haver qualquer potencial conflito de interesses referente a este artigo.
16. Bishop NC, Gleeson M, Nicholas CW, Ali A. Influence of carbohydrate supplementation on plasma cytokine and neutrophil degranulation responses to high intensity intermittent exercise. Int J Sport Nutr Exerc Metab. 2002;12(2):145-56.

7. Green KJ, Croaker SJ, Rowbottom DG. Carbohydrate supplementation and exercise-induced changes in T-lymphocyte function. J Appl Physiol. 2003;95(3):1216-23.

18. Natale VM, Brenner IK, Moldoveanu AI, Vasiliou P, Shek P, Shephard RJ. Effects of three different types of exercise on blood leukocyte count during and following exercise. Sao Paulo Med J. 2003;121(1):9-14.

19. Opstad PK. Alterations in the morning plasma levels of hormones and the endocrine responses to bicycle exercise during prolonged strain. The significance of energy and sleep deprivation. Acta Endocrinol ( $\mathrm{CO}$ penh). 1991;125(1):14-22.

20. Iversen PO, Stokland A, Rolstad B, Benestad HB. Adrenaline-induced leucocytosis: recruitment of blood cells from rat spleen, bone marrow and lymphatics. Eur J Appl Physiol Occup Physiol. 1994;68(3):219-27.

21. Boyum A, Wiik P, Gustavsson E, Veiby OP, Reseland J, Haugen AH, et al. The effect of strenuous exercise, calorie deficiency and sleep deprivation on white blood cells, plasma immunoglobulins and cytokines. Scand J Immunol. 1996;43(2):228-35.

22. Nemet D, Mills PJ, Cooper DM. Effect of intense wrestling exercise on leucocytes and adhesion molecules in adolescent boys. Br J Sports Med. 2004;38(2):154-8.

23. Sellar CM, Syrotuik DG, Field CJ, Bell GJ. The effect of dietary control and carbohydrate supplementation on the immune and hormonal responses to rowing exercise. Appl Physiol Nutr Metab. 2006;31(5):588-96.

24. Lancaster GI, Khan Q, Drysdale PT, Wallace F, Jeukendrup AE, Drayson MT, et al. Effect of prolonged exercise and carbohydrate ingestion on type 1 and type 2 T lymphocyte distribution and intracellular cytokine production in humans. J Appl Physiol. 2005;98(2):565-71.

25. Nieman DC, Davis JM, Henson DA, Walberg-Rankin J, Shute M, Dumke CL, et al. Carbohydrate ingestion influences skeletal muscle cytokine mRNA and plasma cytokine levels after a 3-h run. J Appl Physiol. 2003;94(5):1917-25.

26. Braun WA, Von Duvillard SP. Influence of carbohydrate delivery on the immune response during exercise and recovery from exercise. Nutrition. 2004;20(7-8):645-50

27. Henson DA, Nieman DC, Blodgett AD, Butterworth DE, Utter A, Davis JM, et al. Influence of exercise mode and carbohydrate on the immune response to prolonged exercise. Int J Sport Nutr. 1999;9(2):213-28. 